\title{
El impacto de la COVID-19 en las pequeñas y medianas empresas de comunicación en España
}

\section{The impact of COVID-19 in small and medium-sized communication enterprises in Spain}

Dra. Elena Bandrés-Goldáraz es profesora e investigadora de la Universidad de Zaragoza (España) (bandres@unizar.es) (https://orcid.org/0000-0003-0898-2232)

Mg. Mariola Conde-Casado es profesora e investigadora de la Universidad de Zaragoza (España) (mconde@unizar.es) (https://orcid.org/0000-0003-1348-035X)

Dra. Isabel Iniesta-Alemán es profesora e investigadora en la Universidad Internacional de la Rioja (España) (isabel.iniesta@unir.net) (https://orcid.org/0000-0002-0127-3487)

\begin{abstract}
Resumen
La pandemia provocada por la COVID-19 se ha llevado por delante a buena parte de las pequeñas y medianas empresas españolas. Sin embargo, estas pymes dedicadas a la comunicación han visto cómo la crisis económica no solo no ha afectado de la misma manera que al resto de empresas y autónomos, sino que están saliendo adelante, en una amplia mayoría, con la gran esperanza de que la crisis les respetará en este 2021, a pesar de los duros ajustes que han tenido que hacer ante la reducción mayoritaria de ingresos. El estudio se ha realizado en pymes y autónomos/as de la Comunidad de Aragón fundamentalmente pero también se ha estudiado en una pequeña muestra de las de Madrid, por ser territorios representativos de lo que ocurre en España. El objetivo de este trabajo consiste en averiguar cuáles han sido las medidas adoptadas por estas empresas para afrontar y capear la crisis mundial. A través de una metodología e-Delphi se ha obtenido como principales conclusiones el alto grado de positividad frente a la crisis detectada en estas empresas; sus empeños por salir adelante, a pesar del mazazo económico que ha supuesto para la mayoría la bajada de ingresos y el alto grado de solidaridad para con el resto de la sociedad desplegada por estas personas.
\end{abstract}

\begin{abstract}
The pandemic caused by COVID-19 has taken a large number of small and medium-sized Spanish companies by surprise. However, these SMEs dedicated to communication have seen how the economic crisis has not only not affected in the same way as the rest of companies and self-employed, but they are getting ahead, in a large majority, with the great hope that the crisis will respect them in this 2021, despite the tough adjustments they have had to make before the majority reduction of income. The study has been carried out in SMEs and self-employed workers in the Community of Aragon, mainly, but a small sample of those in Madrid has also been studied, as they are representative of what is happening in Spain. The aim of this work is to find out what measures have been adopted by these companies to face and weather the global crisis.

Through an e-Delphi methodology, the main conclusions obtained were the high degree of positivity in the face of the crisis detected in these companies; their efforts to move forward, despite the economic blow that the drop in income has meant for most of them; and the high degree of solidarity with the rest of society shown by these people.
\end{abstract}

\section{Palabras clave I keywords}

Empresas, pymes, comunicación, COVID-19, gestión de la complejidad, gestión de crisis, resiliencia.

Companies, SMEs, communication, COVID-19, competitive advantage, crisis management, communication, resilience.

Cómo citar: Bandrés-Goldáraz, E., Conde-Casado, M., e Iniesta-Alemán, I. (2021). El impacto de la COVID-19 en las pequeñas y medianas empresas de comunicación en España. Retos Revista de Ciencias de la Administración y Economía, 11(21), pp. 25-40. https://doi.org/10.17163/ret.n21.2021.02 


\section{Introducción}

La crisis económica sin precedentes generada por la COVID-19 en España ha impactado fundamentalmente en la pequeña y mediana empresa. De cada cinco empleos destruidos en 2020, cuatro estaban registrados en organizaciones empresariales con menos de cincuenta personas empleadas.

Según la clasificación de microempresas, pequeña y mediana empresa (PYME) recogida en el Anexo I del Reglamento (UE) nº 651/2014 de la Comisión Europea:

1. La categoría de microempresas, pequeñas y medianas empresas (PYME) está constituida por las empresas que ocupan a menos de 250 personas y cuyo volumen de negocios anual no excede de 50 millones EUR o cuyo balance general anual no excede de 43 millones EUR.

2. En la categoría de las PYME, se define pequeña empresa como una empresa que ocupa a menos de 50 personas y cuyo volumen de negocios anual o cuyo balance general anual no supera los 10 millones EUR.

3. En la categoría de las PYME, se define microempresa como una empresa que ocupa a menos de 10 personas y cuyo volumen de negocios anual o cuyo balance general anual no supera los 2 millones EUR.

Por otro lado, en España también existe una categoría laboral desempeñada por personas cuya labor profesional se define como "autónomo/a". Según el artículo 1 de la Ley 20/2007, de 11 de julio, del Estatuto del Trabajo Autónomo, puede ser catalogado como profesional autónomo o autónoma:

Las personas físicas que realicen de forma habitual, personal, directa, por cuenta propia y fuera del ámbito de dirección y organización de otra persona, una actividad económica o profesional a título lucrativo, den o no ocupación a trabajadores por cuenta ajena. Esta actividad autónoma o por cuenta propia podrá realizarse a tiempo completo o a tiempo parcial.

Atendiendo a los datos de diciembre de 2020, en España están registrados 3271408 autónomos/as que cotizan a la Seguridad Social dentro del Régimen Especial de Trabajadores Autónomos (RETA).

Del volumen total de empresas y de trabajadores, esta investigación se ceñirá a las empresas dedicadas a la gestión integral de la comunicación para organizaciones y personas, que puede contener servicios de consultoría, auditoría de comunicación, imagen y marca corporativa, reputación o relaciones públicas, entre otros campos, una tipificación que se recoge, según la Clasificación Nacional de Actividades Económicas (CNAE), en el grupo $\mathrm{M}$ de dicho ranking, denominado "Actividades Profesionales, Científicas y Técnicas", en el que se recogen las actividades dedicadas a "relaciones públicas y comunicación (...) publicidad; agencias de publicidad; servicios de representación de medios de comunicación; estudio de mercado y realización de encuestas de opinión pública; actividades de diseño especializado y actividades de fotografía”. El estudio se centrará en una representación de las empresas en la Comunidad de Madrid y en la de Aragón, con el fin de acotar el tamaño de la muestra, al mismo tiempo que se obtiene una muestra representativa.

En cuanto al número de empresas inscritas en la Seguridad Social, según el informe elaborado por el Ministerio de Trabajo a diciembre de 2020, las empresas dedicadas a este tipo de actividades en diciembre de 2019 pasaron de 94001 en el conjunto de España, a 92843 en el mismo mes, pero de 2020. En diciembre de 2020, el número de personas registradas en este sector de Relaciones Públicas y Comunicación en todo el país era de 763 867. En el régimen de autónomos, esta misma sección aglutinaba a 
295389 personas. Es esta una tendencia decreciente (Marta-Lazo et al., 2018) que ya se venía experimentando en el sector, aún antes de la crisis actual.

En enero de 2020, en la Comunidad Autónoma de Madrid las personas afiliadas al régimen general en esta actividad fueron 243692 y, en Aragón, 14 510. Estas cifras bajaron en Madrid en diciembre de ese mismo año a 242249 personas, mientras que en Aragón se registró un ligero aumento al subir esta cifra a 14.658. En cuanto al registro de trabajadores autónomos en toda España, en enero de 2020, su número era de 131395 pero terminó el año con una media en diciembre de 295520 afiliados en la categoría M.

En el régimen de trabajadores autónomos en Aragón, se registraron 7703 en enero de 2020 y terminaron el año con un registro de 7737 personas, como puede comprobarse en la Tabla 1.

Tabla 1. Afiliados/as medios del mes y secciones de actividad CNAE 2009

\begin{tabular}{|c|c|c|c|}
\hline & & ENE. 2020 & DIC. 2020 \\
\hline LOCALIZACIÓN & & $\begin{array}{l}\text { SECCIÓN M CNAE } \\
\text { "RELACIONES } \\
\text { PÚBLICAS } \\
\text { Y COMUNICACIÓN" }\end{array}$ & $\begin{array}{l}\text { SECCIÓN M CNAE } \\
\text { "RELACIONES } \\
\text { PÚBLICAS } \\
\text { Y COMUNICACIÓN" }\end{array}$ \\
\hline ESPAÑA & RÉGIMEN GENERAL & 766823 & 763867 \\
\hline C.A. MADRID & RÉGIMEN GENERAL & 243692 & 242249 \\
\hline C.A. ARAGÓN & RÉGIMEN GENERAL & 14510 & 14658 \\
\hline ESPAÑA & AUTÓNOMOS/AS & 131395 & 295389 \\
\hline C.A. MADRID & AUTÓNOMOS/AS & 27015 & 58083 \\
\hline C.A. ARAGÓN & AUTÓNOMOS/AS & 7703 & 7737 \\
\hline
\end{tabular}

Fuente: Elaboración propia

Como es obvia la importancia que ha tenido y tiene la comunicación en cualquier situación y, especialmente, en tiempos de pandemia, se quiere reflejar en este trabajo cómo ha afectado la crisis económica derivada de la crisis sanitaria a las pequeñas y medianas empresas que han hecho y hacen posible este tipo de comunicación en numerosas organizaciones, entidades y empresas, imprescindible para afrontar y superar estos duros momentos.

Ante la imposibilidad de llevar a cabo un escaneo de todas las pymes de comunicación en todo el país se ha realizado un estudio sobre la mayoría de las empresas de comunicación registradas en Aragón y sobre una muestra representativa de las pymes de la Comunidad de Madrid. Se ha elegido Aragón por ser una comunidad que suele utilizarse como referencia media en cuanto a comportamiento político, como ha quedado registrado en el libro "Aragón es nuestro Ohio (2019)", pero también por ser una comunidad de tamaño medio en España.

Este estudio se ha comparado, a grandes rasgos, con los realizados por KPMG y CEOE (2020a) y el realizado por DIRCOM (2020) para las grandes empresas de todos los sectores, para determinar si existen o no coincidencias con las pequeñas y medianas. 


\subsection{La comunicación como herramienta de las empresas al servicio de la sociedad}

El 91,3\% de las grandes empresas contaba con un departamento de comunicación en el año 2010, según se pone de manifiesto en la tercera edición del informe "El estado de la comunicación en España" (2010) publicado por la Asociación de Directivos de Comunicación (DIRCOM), cifra que aumentó hasta alcanzar el 100\% de las empresas en 2018, según datos aportados por esta Asociación en su último informe (2018). El desarrollo de la comunicación está ligado, en las últimas décadas, al ámbito de las grandes empresas (Gómez-Nieto, 2011) ya que cuando se trata de pequeñas y medianas empresas (PYMES), según un informe de la consultora OAK Power publicado por el diario Cinco Días, (2015) este porcentaje desciende hasta el 55\%.

En este marco, el volumen de negocio de las consultoras de comunicación y relaciones públicas aumenta cada año, tanto a nivel internacional como en España, ya que crece la cifra de empresas que entienden que las consultoras especializadas son las organizaciones idóneas para apoyar, en el caso de las grandes corporaciones o, para organizar, en el caso de las pymes, la comunicación integral de sus marcas. En 2019, el $57 \%$ de las compañías afirmaban haber trabajado con consultoras de comunicación. De ellas, el 75\% subrayaba que volverían a trabajar con una y el $88 \%$ las considera como el socio ideal para manejar la estrategia de comunicación y de marca a largo plazo.

Son algunos de los datos que se desprenden del estudio bienal "Agency Scope 2019/2020" elaborado por la consultora internacional Scopen (2020), donde se asegura que las empresas de comunicación y relaciones públicas contribuyen en un $16 \%$ al crecimiento del negocio de sus clientes. En España constituyen este sector agencias de perfil independiente y de muy pequeño tamaño que suelen trabajar para las pymes, frente a grandes multinacionales, que son más atractivas y competitivas para las grandes empresas (AxiCom, 2015). Los cambios vertiginosos a los que tienen que hacer frente el sector han provocado también la aparición de empresas colaborativas, formadas por autónomos/as especialistas en áreas concretas de la comunicación que ofrecen sus servicios de forma independiente pero englobados en una marca común.

Este nuevo entorno, inmerso en la eclosión de nuevos modelos de negocio y marcado por la presencia de la Tecnología de Inteligencia, requiere que las empresas se diseñen con base en estructuras flexibles, eficientes y dinámicas (Ravina-Ripoll et al., 2019). Para conseguir esto es imprescindible que las personas que forman el equipo de las nuevas empresas de comunicación y relaciones públicas acepten con naturalidad y sin temor (Foncubierta-Rodríguez \& Sánchez-Montero, 2019) el nuevo contexto de la comunicación digital.

Como recogen Gómez y Bandrés (2014, p. 144), "las agencias de comunicación ofrecen servicios a aquellas organizaciones que, por su dimensión, no pueden disponer de un gabinete propio, pero sí son conscientes de lo impredecible que resultan hoy los servicios de comunicación”. Siguiendo a Marín-Dueñas (2015) las pymes se plantean la necesidad de invertir en comunicación ya que la consideran como un factor de éxito que favorece los buenos resultados, así como la buena marcha de la organización al ser una herramienta imprescindible para relacionarse con sus públicos de interés ya sean internos o externos. Para este autor, las pymes entienden que la comunicación estratégica debe ser gestionada por personal especializado y profesional, por lo que, cuando no disponen de presupuesto para incorporar a un especialista en plantilla, externalizan el servicio a consultoras de comunicación.

El informe de Scopen asegura que cada vez más empresas son conscientes de la necesidad de contar con las consultoras de comunicación o agencias de relaciones públicas que les ayuden a gestionar su imagen y reputación. El conocimiento del mer- 
cado, el cliente y la marca (37\%), proactividad e iniciativa (37\%) y creatividad e ideas innovadoras $(31 \%)$, son las características más valoradas por las empresas anunciantes en una consultora de comunicación, según Scopen.

En esta misma línea, un informe realizado por la revista especializada El Publicista (2019) destaca que las empresas españolas valoran de forma muy positiva tres aspectos clave de las agencias de comunicación: su capacidad estratégica para generar negocio desde la gestión de la comunicación (es un aspecto clave para ocho de cada diez entrevistados), su capacidad de aportar una visión innovadora y creativa para llegar a la audiencia $(70 \%)$ y el que sean interlocutoras perfectas entre la empresa y sus grupos de interés.

Una de las evidencias que ha dejado la pandemia en el ámbito económico y social ha sido el refuerzo de la implicación de las organizaciones con sus grupos de interés, en el ámbito de responsabilidad social de su actividad. Entre el 14 de marzo de 2020 (el día anterior a la entrada en vigor en España del Estado de Alarma) y el 14 de abril de ese mismo año, según un informe de la consultora CANVAS (2020) se han identificado vinculadas a la COVID-19, más de 650 iniciativas empresariales y más de 9000 impactos en medios de comunicación protagonizados por las grandes empresas, pymes y autónomos/as. Algunas de estas iniciativas han sido donaciones, acciones solidarias o adaptaciones de los modelos de producción dirigidas a todos los grupos de interés, desde empleados a clientes y proveedores, hasta la sociedad en general, en especial los sectores más necesitados o vulnerables, como se recogerá más adelante.

Según este informe, un $41 \%$ de las iniciativas se han enfocado mayoritariamente en el conjunto de la sociedad en general, poniendo en evidencia la fuerza que pueden manifestar las empresas al reaccionar ante situaciones urgentes, así como su capacidad para ayudar a los sectores más débiles.

Ante esta pandemia sanitaria y social, más del 39\% de las acciones solidarias de las empresas han puesto el foco en la salud y el bienestar de sus grupos de interés más cercanos, tanto externos como internos, tal y como se constata en la Tabla 2:

Tabla 2. Iniciativas empresariales frente a la COVID-19

\section{TIPOLOGÍA DE INICIATIVAS}

\section{CORPORATIVO}

\section{EMPLEADOS}

- Publicidad específica sobre COVID-19

- Ajustes de costes, reducción o reajuste de actividad

- Suspensión de actividades de marketing o publicidad

- Mensajes públicos de CEO o alta dirección

- Recorte de sueldos de directivos

- Suspención de dividendo y/o aplazamiento de compra de acciones

- Cancelación o celebración telemática de juntas de accionistas

- Lanzamiento de planes post COVID-19

\section{CLIENTES}

- Mantenimiento de actividad y del empleo

- Bonus extra para empleados que tranajen durante el estado de • alarma

- Medidas relacionadas con salud, seguridad y bienestar (incluye teletrabajo)

- Nuevas contrataciones o refuerzo de turnos

- Voluntariado corporativo o iniciativas promovidas por los empleados
- Lanzamiento o redefinición de servicios

- Medidas reforzadas de seguridad y prevención

- Priorización de ciertos colectivos en la prestación del servicio

- Aplazamiento, retrasos o moratoria de pagos a clientes

- Prestación de servicios gratuitos para colectivos desfavorecidos 


\begin{tabular}{|l|l|}
\hline \multicolumn{1}{|c|}{ SOCIEDAD } & \multicolumn{1}{|c|}{ PROVEEDORES } \\
\hline - Donación económica & $\begin{array}{l}\text { Adelantamiento de pa- } \\
\text { goss a proveedores }\end{array}$ \\
- $\begin{array}{l}\text { Adaptación temporal a la producción, servicios o personal a } \\
\text { las necesidades surgidas por la crisis }\end{array}$ & \\
- Adhesión a plataformas o iniciativas o alianzas con entidades & \\
- Acciales & \\
- Acompañamiento a colectivos vulnerables & \\
- Iniciativas culturales o formativas & \\
- Apoyo a la innovación & \\
\hline
\end{tabular}

Fuente: Canvas, 2020

A partir de este listado general de iniciativas, destacan las donaciones en especie, en concreto y especialmente de material sanitario como mascarillas y geles hidro-alcohólicos de desinfección de manos, así como de alimentos y otros dispositivos electrónicos. También se reseñan las acciones directamente monetarias que, en conjunto, representan más del 19\% del total. En cuanto a las iniciativas con el personal destacan el mantenimiento de la actividad y del empleo y el refuerzo de las medidas de salud, seguridad y bienestar. El estudio de CANVAS (2020) destaca, además, las acciones relativas al establecimiento de alianzas, adhesión a iniciativas o plataformas sociales, algo que denota un nivel alto de compromiso con el entorno, con vocación de permanencia y con un fuerte componente de cooperación.

Entre las conclusiones de este informe se pone de manifiesto que esta crisis ha fortalecido el rol y el protagonismo de las personas dentro de las empresas. De esta manera, plantea la necesidad de consolidar el clima ético generado frente a la crisis reforzando la sostenibilidad, la resiliencia organizacional y la flexibilidad como los vectores clave de la nueva cultura empresarial para adaptarse al nuevo escenario pos-COVID.

Una macro iniciativa empresarial puesta en marcha por una conocida marca de supermercados promovió, junto con sus doscientas empresas proveedoras, la plataforma \#EstoNOtienequePARAR, para reconocer públicamente el esfuerzo de las empresas por seguir trabajando y generar un clima de confianza, fundamentalmente a nivel interno. Salió a la red el 16 de marzo de 2020 para unir y animar, tal y como lo recogen en su web, "a quienes trabajaban garantizando los suministros esenciales pese a las grandes dificultades”. A través de dicha iniciativa se:

Alentaba a todas las empresas, emprendedores y autónomos que no podían trabajar o abrir su negocio a que continuasen activos, inspirándoles con casos de éxito reales que sucedían en aquel momento para que también innovaran, reinventaran su negocio o se readaptaran a la nueva situación.

En pocos meses alcanzó más de 3000 adhesiones y, tras finalizar el confinamiento a partir del 21 de junio de 2020, fecha en la que se dio por concluido el Estado de Alarma que decretó el total confinamiento domiciliario, salvo para sectores de primera necesidad, esta iniciativa dio paso a una segunda fase llamada \#EActíVate, con el objetivo de "acelerar y activar nuestra economía para superar las consecuencias sociales y económicas del impacto que la crisis sanitaria está teniendo en nuestra estructura empresarial e industrial y nuestra economía”. 
Se dieron a conocer más de 3000 casos reales de empresas de todos los tamaños, trabajadores autónomos/as de todo el estado español. En Aragón también suscitó numerosas adhesiones. Tal y como recogen en su página web, la difusión de estas experiencias se dio a conocer por diferentes canales en los que pudieron verse una ingente cantidad de noticias y que posibilitaron la retroalimentación por parte del público, tal y como recogen en su web: "más de 100 casos publicados en televisiones/radios nacionales y autonómicas; 800 noticias en prensa; 640 casos explicados en la web y 700000 visualizaciones de los vídeos de las empresas en nuestros canales".

Además de este proyecto, en la Comunidad de Aragón se pusieron en marcha iniciativas como:

- \#AragonenMarcha, gestionada por CEOE Aragón en la que se centralizaron donaciones económicas, de productos o servicios junto con el resto de agentes sociales y el Gobierno de Aragón.

- \#LasMarcasSeSalen. Con este "hashtag", se llevó a cabo una campaña de comunicación para rendir un homenaje a las 230 marcas que se sumaron a esta iniciativa solidaria. El objetivo fue generar confianza en el futuro y fortalecer los lazos trazados entre empresas y personas para recuperar la "nueva normalidad" en la que se instaló Aragón y el resto de España, tras la finalización del confinamiento domiciliario.

\section{Metodología y muestra}

La necesidad de obtener información sobre el impacto generado por la crisis sanitaria en la actividad de las pymes de comunicación, prácticamente al mismo tiempo que se sigue sufriendo la pandemia, ha obligado a adoptar unos parámetros de análisis basados en la versatilidad de la metodología e-Delphi, definida por Lindqvist y Nordänger (como se cita en Mamaqi et al., 2010) "como un tipo especial de investigación en la que se utilizan cuestionarios para la recogida de datos", y en la que "en los últimos años, el uso del correo electrónico se ha hecho cada vez más frecuente para llevar a cabo el proceso".

Se ha seguido el protocolo de fases, recogidas por Reguant y Torrado (2016, p. 92) iniciado por la consulta a tres expertas que han ayudado a validar los criterios de las preguntas, así como su formulación. Se ha establecido una encuesta de conveniencia dirigida a estas empresas para obtener información específica a través de un formulario descriptivo, con preguntas cuantitativas de fase básica y otras preguntas cualitativas para lograr la información sobre respuestas personales ante problemas generales, provocados por la COVID-19. También se ha contactado con las empresas a través del correo electrónico y la red social de LinkedIn para explicarles la colaboración con el proyecto y se ha obtenido de ellas opiniones muy valiosas sobre temas específicos que no se contemplaban en el cuestionario.

La encuesta realizada consta de seis primeras preguntas en las que se determina el sexo, la edad, tamaño de la empresa, estudios de la persona dirigente que contesta, domicilio de la empresa y años de creación de la empresa.

El cuestionario contiene otras diez preguntas, en las que se plantea cómo ha afectado la COVID a la empresa; si se ha acogido a un erte ${ }^{1}$ y si se está, en el momento

1 Erte, definido por el Diccionario Panhispánico del Español Jurídico, es el "procedimiento para la suspensión del contrato de trabajo o la reducción de la jornada laboral, de carácter temporal, por 
de contestar, en uno. Se pregunta también sobre cuál de las medidas sugeridas se ha tomado principalmente para adaptarse a la situación generada por la COVID-19 (teletrabajo; reducción de plantilla; solicitud de crédito financiero; focalización del trabajo en internet y redes sociales; cambio de actividad u otra medida). La pregunta 5 es de respuesta abierta para quienes hayan contestado "Otra medida". La 6 solicita que diga si la empresa ha llevado a cabo alguna iniciativa solidaria durante la pandemia. La 7 solicita que, en caso de haberlo hecho, indique cuál o cuáles han sido. La 8 solicita que se informe sobre cuánto facturaba la empresa al año, antes de la COVID. La penúltima, cuál es la facturación a finales de 2020 y, por último, se pregunta si cree que la empresa podrá aguantar abierta en 2021.

\subsection{Tipología de la muestra}

Para localizar a las empresas aragonesas, se ha utilizado el listado recogido en la agenda de la Asociación de Periodistas de Aragón, así como el directorio de la Asociación de Directivos de Comunicación (DIRCOM-Aragón) de dicha Comunidad Autónoma.

Con el objeto de encontrar a las empresas madrileñas para este trabajo se realizó un cribado de las empresas de comunicación integradas en el ranking de empresas del sector Relaciones públicas y comunicación de la web "El Economista.es" en el que aparecen las empresas ordenadas por su cifra de ventas. Esta información procede, según la propia página:

De la Base de Datos de INFORMA D\&B S.A.U. (S.M.E.), que se nutre de múltiples fuentes de información, públicas y privadas como el BORME (Boletín Oficial del Registro Mercantil), Depósitos de Cuentas Oficiales, BOE (Boletín Oficial del Estado), Boletines Oficiales Provinciales y de CC.AA., Prensa nacional y regional, Investigaciones ad hoc y Publicaciones Diversas.

El buscador permite discriminar el rango de búsqueda por tamaño y ciudad por lo que se ha utilizado este método para encontrar las primeras veinte pequeñas empresas y las primeras veinte, de tamaño medio.

En total se envió el cuestionario a 50 empresas entre el 27 de diciembre de 2019 y el 22 de enero de 2021, de las que contestaron 32. Teniendo en cuenta que el método Delphi, (independientemente de su variante electrónica) considera adecuado el número de 30 personas expertas para recabar la información sobre el asunto a investigar, se estaría dentro de los parámetros muestrales óptimos para recabar los datos. También se ha obtenido más información a través del contacto directo con varios profesionales que han valorado de manera particular la situación actual.

\section{Resultados}

De los resultados obtenidos se extrae que el 65,6\% es mujer, frente al 34,4\% que es varón. El 50\% se encuentra entre los 41 y 50 años. El 25\%, entre los 51 y 60 . El 12,5\% entre 61 y 65 años. El 3,1\% tiene más de 66 años. Otro 3,1\%, entre los 20 y 30 años de edad. El 6,3\%, entre 31 y 40 años.

En cuanto al tamaño de la empresa, el $43,8 \%$ tiene entre dos y cinco trabajadores. El 34,4\% es autónomo/a. El 12,5\%, entre seis y diez. El 6,3\%, entre 11 y 20 y el 3,1\%, más de 51 trabajadores. Respecto a los estudios, el 43,8\% tienen título universitario en

causas económicas, organizativas o de producción o derivadas de fuerza mayor. Una figura del derecho laboral regulada en el Real Decreto 1483/2012, de 29 de octubre. 
Periodismo, Comunicación o Publicidad. El 53,1\%, su grado o licenciatura proviene de otros estudios universitarios y el 3,1\%, de otros grados.

El 53,1\% de las empresas consultadas se encuentran en Zaragoza, el 31,3\% en Madrid, el 9,4\%, en Huesca y el 6,3\%, en Teruel. El 30,4\% creó la empresa hace más de 20 años. El 43,5\%, hace diez. El 17,4\%, hace cinco. El 4,3\%, hace uno y, otro 4,3\% hace menos de un año, como puede verse de manera global en la Figura 1.

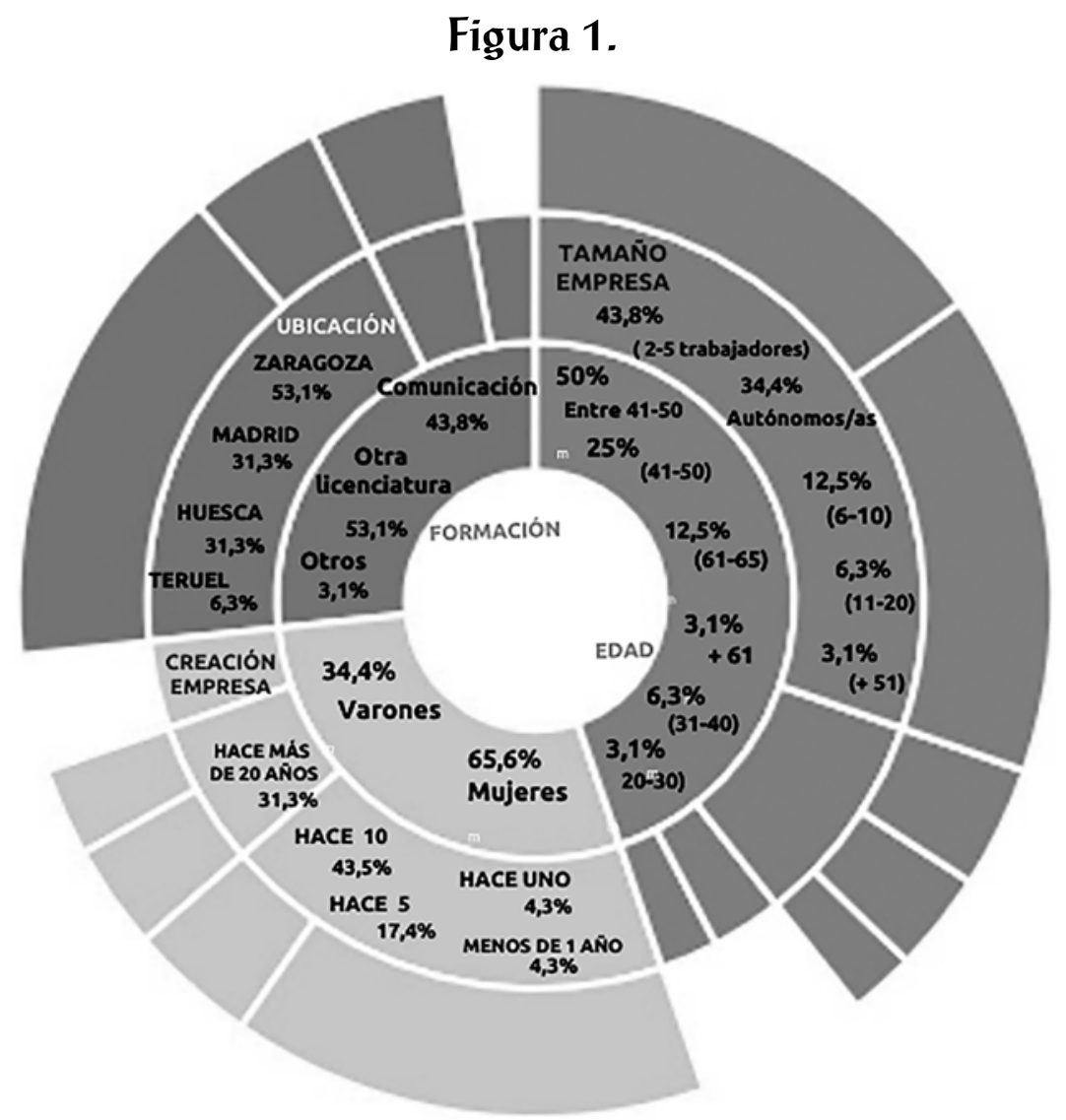

En la Tabla 3 se muestran las respuestas a las preguntas 1. ¿Cómo ha afectado la COVID-19 a tu empresa? 2. ¿Te has acogido a un erte? 3. ¿Estás en un erte en estos momentos? 4. ¿Cuál de estas medidas has tomado principalmente para adaptarte a la situación generada por la COVID-19?

Tabla 3. Respuestas a las preguntas 1, 2, 3 y 4

\begin{tabular}{|c|c|c|c|}
\hline (1) & $(2)$ & (3) & $(4)$ \\
\hline $\begin{array}{l}\text { ¿Cómo ha afectado en } \\
\text { COVID a tu empresa ? }\end{array}$ & $\begin{array}{l}\text { ¿Te has } \\
\text { acogido } \\
\text { a un erte? }\end{array}$ & $\begin{array}{l}\text { ¿Estás en erte en } \\
\text { estos momentos? }\end{array}$ & $\begin{array}{c}\text { ¿Cuál de estas medidas } \\
\text { has tomado para } \\
\text { adaptarte? }\end{array}$ \\
\hline $\begin{array}{c}\text { Reducción de trabajo } \\
25 \% \quad 28 \%\end{array}$ & $21,9 \% \mathrm{SI}$ & $93,7 \% \mathrm{NO}$ & Teletrabajo $62,4 \%$ \\
\hline $\begin{array}{c}\text { Reducción de trabajo } \\
50 \% \quad 18,1 \%\end{array}$ & $78,1 \%$ NO & $6,3 \% \mathrm{SI}$ & Internet y RSS $18,8 \%$ \\
\hline $\begin{array}{c}\text { Reducción de trabajo } \\
75 \% \quad 3,1 \%\end{array}$ & & & Créditos $6,3 \%$ \\
\hline Cerrado 9,4\% & & & Reducción de plantilla 3, $1 \%$ \\
\hline No me ha afectado $21,9 \%$ & & & Cambio de aactividad 3,1\% \\
\hline He aumentado el trabajo $19,4 \%$ & & & Otras medidas $6,3 \%$ \\
\hline
\end{tabular}


Mientras que las respuestas a la pregunta 5. Si has elegido "otra medida" en la pregunta anterior, indica, por favor, cuáles son, las respuestas mayoritarias han sido, tal y como se puede ver en la Tabla 4:

Tabla 4.

\begin{tabular}{|l|}
\hline Flexibilidad \\
\hline Teletrabajo y crédito ICO \\
\hline Cerrar la empresa varios meses \\
\hline Créditos financieros \\
\hline $\begin{array}{l}\text { Hemos cerrado una de las cabeceras, por lo que se ha reducido la plantilla, y además hicimos } \\
\text { teletrabajo durante el confinamiento }\end{array}$ \\
\hline
\end{tabular}

Fuente: Elaboración propia

Y en la Tabla 5 se recogen las respuestas a las preguntas: 6. ¿Como empresa, has llevado a cabo alguna iniciativa solidaria durante la pandemia? 8. ¿Cuánto facturaba tu empresa al año, antes de la Covid-19? 9. ¿Cuál es tu facturación a finales de 2020 respecto a 2019? y 10. ¿Crees que tu empresa podrá aguantar abierta en 2021?

Tabla 5. Respuestas a las preguntas 6, 8, 9 y 10

\begin{tabular}{|c|c|c|c|}
\hline (6) & (8) & (9) & (10) \\
\hline $\begin{array}{c}\text { ¿Alguna iniciativa } \\
\text { solidaria en la pandemia ? }\end{array}$ & $\begin{array}{c}\text { Facturación empresas } \\
\text { en } 2019\end{array}$ & $\begin{array}{c}\text { Facturación } \\
\text { empresas en } \\
2020 \text { respecto al } \\
2019\end{array}$ & $\begin{array}{l}\text { ¿Aguantará tu } \\
\text { empresa en } \\
2021 ?\end{array}$ \\
\hline $45,2 \% \mathrm{Si}$ & $\begin{array}{c}\text { Entre } 300.000 \text { y } 400.000 € \\
21,8 \%\end{array}$ & $62,6 \%$ Inferior & $68,7 \% \mathrm{Si}$ \\
\hline $56,6 \%$ No & $\begin{array}{c}\text { Entre } 15.000 \text { y } 30.000 € \\
18,8 \%\end{array}$ & $15,6 \%$ Igual & $6,3 \% \mathrm{No}$ \\
\hline \multirow[t]{6}{*}{$3,2 \%$ No lo sabe } & $\begin{array}{c}\text { Entre } 30.0001 \text { y } 50.000 € \\
18,8 \%\end{array}$ & $21,8 \%$ Superior & $25 \%$ No lo sabe \\
\hline & $\begin{array}{c}\text { Entre } 50.001 \text { y } 100.000 € \\
3,1 \%\end{array}$ & & \\
\hline & $\begin{array}{c}\text { Entre } 100.001 \text { y } 200.000 € \\
11,5 \%\end{array}$ & & \\
\hline & $\begin{array}{c}\text { Entre } 200.001 \text { y } 300.000 € \\
10,5 \%\end{array}$ & & \\
\hline & $\begin{array}{c}\text { Más de } 400.000 € \\
9,2 \%\end{array}$ & & \\
\hline & $\begin{array}{c}\text { Menos de } 15.000 € \\
6,3 \%\end{array}$ & & \\
\hline
\end{tabular}

La pregunta número 7 solicitaba que, en caso de haber llevado a cabo alguna acción solidaria, indicaran por favor cuál o cuáles habían sido. Las respuestas obtenidas fueron:

- Apoyo a clientes con problemas, ONG, etc. dentro de nuestro ámbito de actuación

- Creación y Producción de un vídeo motivador para resto de personas y empresas. 
- Ofrecer a mis clientes habituales un plan de comunicación específico para ayudarles, pero al final no ha servido de nada y he cerrado.

- Varias campañas. Para recoger fondos. Difundir la labor de otros. Y para contagiar la confianza que necesitamos para salir de esta crisis sanitaria y económica.

- Estamos en proceso ya que la pandemia no ha acabado.

- Varias con IFEMA.

- Colaboración con la ONG Bokatas Zaragoza.

- Adhesión a iniciativas solidarias como la realizada por CEOE.

- Difusión oferta hostelera.

- Donamos dinero e hicimos acopio de material para la elaboración de mascarillas.

- Ayudar a comunicar ideas solidarias de empresas.

- Apoyo a diferentes ONG que están dentro del programa del Grupo.

- Hemos donado espacio publicitario para poner en valor todas las iniciativas solidarias de la zona, que han sido muchas, con el objetivo de conseguir nuevos colaboradores para las mismas.

\section{Discusión y conclusiones}

Puede decirse que los efectos de la crisis económica generados por la COVID-19 en las empresas de comunicación y trabajadores autónomos estudiados no han sido tan duros como el resto de las pequeñas y medianas empresas ya que, a pesar de la dramática situación han logrado salir a flote en este año, aunque con unas pérdidas considerables respecto a su situación de antes de la pandemia.

La mitad ha tenido que reducir su trabajo entre un 25 y un $75 \%$; casi un $10 \%$ han cerrado, mientras que a cerca del $22 \%$ no le ha afectado la crisis y el $18,8 \%$ ha aumentado su trabajo en tiempos de crisis.

Cerca de un $22 \%$ se han acogido a un erte pero en el momento de contestar la encuesta, el 93,7\% no lo estaba. Las empresas han capeado la mala situación aplicando medidas como el Teletrabajo (62,5\%); volcando su actividad en Internet y en las redes sociales $(18,8 \%)$; mientras que un $6,3 \%$ ha solicitado créditos y otros han reducido la plantilla o han cambiado de actividad, con un 3,1\% respectivamente. Otras de las medidas adoptadas han sido la "flexibilidad", unir el teletrabajo y la solicitud de un crédito ICO; cerrar la empresa varios meses; reducir la plantilla y teletrabajar.

La solidaridad es otro rasgo identificativo de estas empresas en las que un 45,2\% ha desarrollado alguna iniciativa solidaria como entidad, a pesar de que la facturación en 2020 ha sido inferior a la de 2019 para un 62,5\%. De las personas que han sido solidarias, el $85,7 \%$ ha llevado a cabo iniciativas a pesar de que su facturación en 2020 ha sido inferior al año anterior y, el resto presentaba una cuenta de resultados igual al del año anterior.

A pesar de la mala situación generada por la crisis sanitaria, el 68,8\% cree que su empresa aguantará en 2021, frente al 6,3\% que considera que no lo hará. Un 25\% no lo sabe.

Respecto a estos datos, las empresas españolas de todos los sectores durante la pandemia, y especialmente en el Estado de Alarma, han empeorado su valoración de la situación económica y de sus previsiones a futuro. El indicador de confianza empresarial ha sido, desde el primer trimestre, el valor más bajo de la serie histórica (95,5 puntos), según el INE (2020). En este entorno desfavorable han sido muchas las compañías, especialmente pequeñas y medianas, las que se han visto obligadas a presentar expedientes de regulación temporal de empleo. Muchas empresas han sufrido una caída brusca de su facturación, así como de las inversiones que tenían previstas. 
En el balance de situación sobre las empresas españolas, que de forma periódica realizan KPMG y CEOE (2020b), las personas directivas revelan que se han visto obligadas por la pandemia a adoptar medidas de forma rápida y más allá de sus planes estratégicos, con un doble objetivo. Por un lado, garantizar la salud y el bienestar de sus trabajadores y trabajadoras, proveedores y clientes, con el fin de contribuir con su responsabilidad a frenar la expansión del virus y, por el otro, mantener en la medida de lo posible su actividad, intentando contener los aspectos adversos que el coronavirus implica para su trabajo diario. Objetivos que coinciden con las actitudes reflejadas en las respuestas obtenidas de las pequeñas y medianas empresas encuestadas.

Las empresas reflejadas en el informe anterior han visto afectados todos sus ámbitos de actividad y sus sistemas de funcionamiento por la COVID y también por las medidas del Gobierno para combatirla, tal y como puede apreciarse en la Figura 2.

\section{Figura 2. Áreas de las empresas que se ven afectadas por el coronavirus}

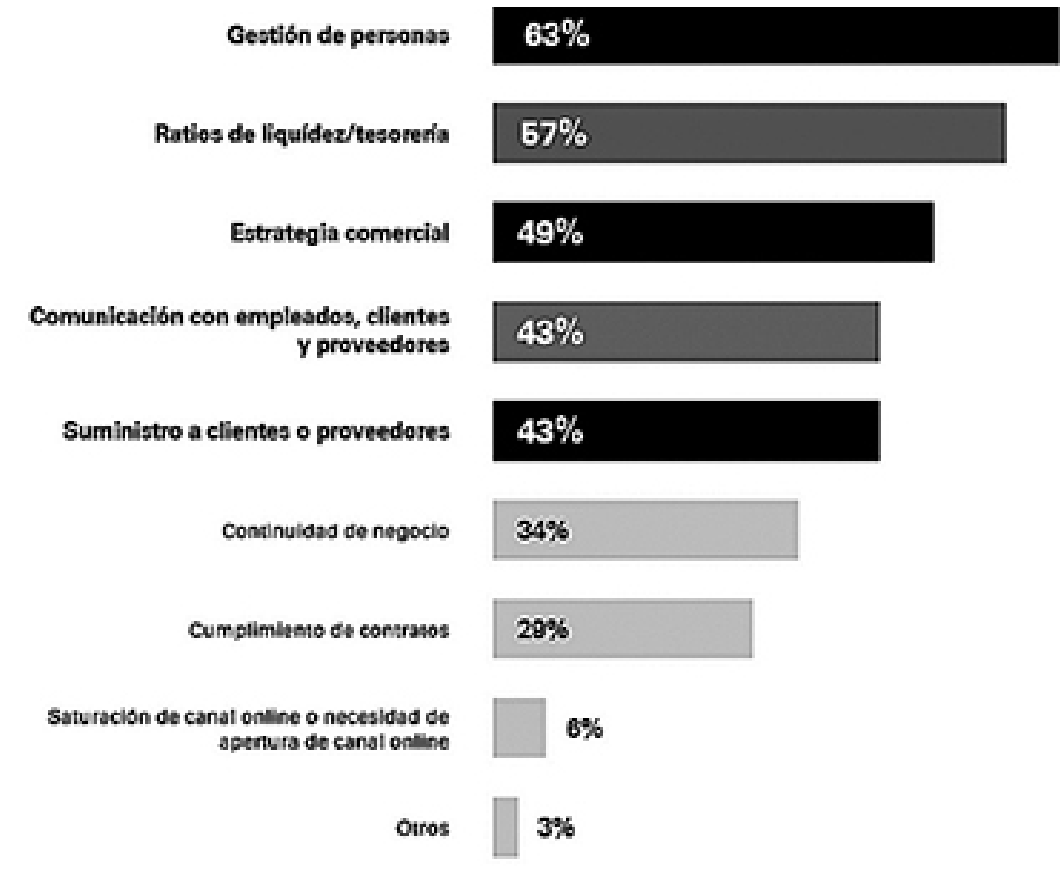

Fuente: KPMG y CEOE, 2020

Las áreas funcionales más afectadas por la emergencia sanitaria han sido la gestión de personas, la tesorería y la gestión comercial. Ante esta crisis, las empresas grandes han aplicado diferentes medidas entre las que destaca también el teletrabajo que ha adoptado la gran mayoría de las pymes consultadas, hasta alcanzar el $96 \%$.

También, se han modificado las estrategias de marketing y comerciales y, entre otras cosas, se han eliminado los viajes comerciales y los llevados a cabo por el personal, además de suspenderse eventos, tanto internos como externos. Asimismo, se han modificado los protocolos y sistemas de comunicación de las empresas con sus grupos de interés y se ha agilizado la transformación digital de las compañías, así como su comunicación digital, con la apertura de nuevos canales on line, en el entorno de la web 2.0.

Para las personas encuestadas que ocupan un puesto directivo, la actual situación ha venido para quedarse y va a transformar algunos aspectos del negocio a largo plazo. La comunicación, la digitalización, las formas de trabajo y la gestión de riesgos son los ámbitos más sensibles a estos cambios, tal y como puede apreciarse. 
La prolongación de la pandemia, a la espera de que la administración de las vacunas sea una realidad en todas las sociedades, ha hecho que se sigan manteniendo en el tiempo estas medidas, tal y como se recoge en la Figura 3.

\section{Figura 3. Evolución de las medidas adoptadas por las empresas ante el coronavirus}



Fuente: KPMG y CEOE, 2020

No obstante, según se puede observar, si se realiza una comparativa entre el primer y el último informe de KPMG y CEOE (2020a y 2020b), la valoración de la evolución de la economía para después de la crisis COVID es levemente más optimista que en pleno confinamiento, algo que coincide con las previsiones de las pequeñas y medianas empresas, así como trabajadores autónomos/as.

Teniendo en cuenta esta comparativa, se puede observar que es precisamente la comunicación y la gestión de personas el principal reto al que han tenido, y tendrán que hacer frente las empresas ante la crisis COVID. La transformación digital también es un área primordial para la mayoría de los empresarios/as, así como la mejora de procesos y otras medidas de reestructuración operativa.

Para DIRCOM a nivel estatal (2020), la comunicación corporativa se ha revelado durante esta crisis como una herramienta esencial en la sociedad en torno a una triple función: 
1. Como herramienta para informar con rigor y claridad y para concienciar con sosiego a la población sobre la situación sanitaria y social derivada de la COVID. En sus orientaciones a los países sobre la pandemia, la Organización Mundial de la Salud apunta que una comunicación oportuna y transparente, con información correcta y basada en evidencias, pero también honesta y franca, con empatía y comprensión por las preocupaciones del público, es fundamental para que las personas conozcan los riesgos de la COVID-19 y sigan las recomendaciones de las autoridades para proteger su salud y la de su entorno.

En este contexto, los hábitos y tendencias de consumo se han visto drásticamente modificados. Los diarios digitales han recibido un $45 \%$ más de páginas vistas y han incrementado su tráfico un 100\%. La audiencia digital de la radio on line ha crecido en un $112 \%$ (por lo que se ha posicionado como uno de los medios que da más confianza a la audiencia) y la televisión on line en directo ha sumado un $93 \%$ de personas usuarias únicas. Por su parte, la televisión se ha convertido también en la fuente principal de entretenimiento e información y los informativos han aumentado en el $65 \%$ en su consumo diario, como recoge MontañaBlasco et al. (2020).

2. Como herramienta de comunicación interna para aumentar el sentimiento de pertenencia del personal contratado en plantilla y cuidarles desde el punto de vista de su salud física y emocional. En el tiempo de pandemia, prácticamente el $100 \%$ de las compañías, consultadas por DIRCOM, establecieron protocolos de seguridad y protección para sus empleados y empleadas, que se tuvieron que implementar y, a la vez, comunicar.

La multicanalidad ha sido factor común en los protocolos de comunicación interna. Apps, podcasts, redes sociales internas, grupos de WhatsApp, teléfono de atención al empleado/a... Según DIRCOM, las empresas han diversificado sus canales de comunicación con su personal y han reactivado algunos a los que venían dando poco uso. El reto ha sido crear una nueva narrativa de comunicación interna, para los que se han utilizado nuevos contenidos con diferentes objetivos en las que se han utilizado fuentes creíbles y en las que se han ofrecido información rigurosa y veraz.

3. Como herramienta al servicio de la sociedad, para que las empresas puedan transmitir su propósito y sus retos futuros en materia de empleo, sostenibilidad o responsabilidad social. Durante la pandemia, además, la mayoría de las marcas comunicaron su solidaridad con la situación, a través de campañas enfocadas en el propósito corporativo, mientras rebajaban la comunicación comercial de sus productos. Para ello, se intensificaron las acciones de comunicación externa multicanal dirigidas a comunicar a la sociedad las medidas y acciones que desde la propia empresa se pusieron en marcha para contribuir a mejorar la situación, tal y como se ha referenciado.

En este ámbito, según un informe de Deloitte (2020), casi cuatro de cada cinco personas podrían citar un momento en el que una marca respondió positivamente a la pandemia y, una de cada cinco, estuvo muy de acuerdo en que llevó a una mayor lealtad a la marca de su parte. Por el contrario, más del 25\% de los que notaron que las marcas actuaban en su propio interés se alejaron de esas marcas.

En general, el comportamiento empresarial de las pequeñas y medianas empresas de comunicación, junto al de las personas autónomas que trabajan en este sector, en plena crisis generada por la COVID-19 ha logrado adaptarse, en su gran mayoría, a las 
durísimas consecuencias generadas por el cese de la actividad económica en buena parte del sector empresarial.

Si se tienen en cuenta los estudios realizados por KPMG y CEOE (2020b), en los que se analiza la situación de la empresa española ante la COVID-19, junto con el análisis de DIRCOM sobre comunicación en tiempos de pandemia, citados con anterioridad, y se comparan con los resultados que arroja la encuesta de esta investigación, se deduce que las pymes y autónomos de empresas de marketing y comunicación en nuestro país se han visto beneficiadas por el aumento de las necesidades de las empresas de comunicar sobre su empresa y su actividad provocado de forma generalizada por la irrupción de la COVID-19.

La casi totalidad de las empresas españolas han visto trastocado su modelo de negocio y han tenido que adaptarse de forma rápida a una nueva situación organizacional complicada y altamente cambiante y flexible en un entorno incierto. Ante esta situación, estas organizaciones han puesto en marcha o han acelerado su proceso de transformación digital y sus modelos de comunicación, tanto en el ámbito interno como en el externo. Asimismo, en una situación límite como la que se ha vivido en el marco de esta pandemia, las empresas han potenciado su línea de responsabilidad social y ayuda al entorno.

La información en el interior de las empresas se ha hecho más necesaria que nunca, no solo para la comunicación de nuevas situaciones laborales sobrevenidas con la pandemia, sino para poner en marcha y organizar los nuevos procesos de trabajo a distancia, el establecimiento ordenado de los flujos de información o las nuevas formas de conciliación y relación con todos los miembros de la organización, entre otras situaciones. De igual manera, la comunicación con el exterior, tanto con proveedores como con clientes, tanto con administraciones públicas como con medios de comunicación, ha dado un giro rápido y radical y las empresas han tenido que adaptarse a nuevas herramientas 2.0, nuevas fórmulas de venta, diálogo y relación con sus grupos de interés.

Todos estos cambios impactan de lleno en el área de comunicación que es la encargada de la comunicación interna, junto al departamento de recursos humanos, de la comunicación con proveedores y clientes, junto al departamento de marketing y, de la línea de la responsabilidad social corporativa (RSC) de la organización. Este aumento de trabajo en los departamentos de comunicación de las grandes empresas y el surgimiento de nuevas necesidades comunicacionales en las pymes ha provocado un incremento de la demanda de servicios de consultoría, asesoría y trabajos de comunicación que ha beneficiado a las pymes y personas autónomas especializadas en esta materia, con independencia del momento de incertidumbre y de crisis generalizada que se ha sentido con mayor virulencia en otros sectores del tejido empresarial.

\section{Referencias}

AxiCom (2015). Nuevos Retos y Oportunidades de los Departamentos de Comunicación en España. https://bit.ly/36gDWdn

Afiliados medios del mes por CC. AA, provincias y secciones de actividad CNAE 2009. Régimen general (diciembre 2020). https://bit.ly/37RYppV

Afiliados medios del mes por CC. AA, provincias y secciones de actividad CNAE 2009. Régimen especial de trabajadores autónomos (diciembre 2020). https://bit.ly/37RYppV

Afiliados medios del mes por CC. AA, provincias y secciones de actividad CNAE 2009. Régimen general (enero 2020) https://bit.ly/37RYppV

Afiliados medios del mes por CC. AA, provincias y secciones de actividad CNAE 2009. Régimen especial de trabajadores autónomos (enero 2020). https://bit.ly/3ku9ORK 
CANVAS Estrategias Sostenibles (2020). Ética empresarial y Agenda 2030 en tiempos de COVID-19. El compromiso de las empresas durante la pandemia. https://bit.ly/3iJRdQY

Cinco Días (27 de abril de 2015). https://bit.ly/2LTzeLU

Deloitte (2020). 2021 Global Marketing Trends. https://bit.ly/2Y7GTsr

DIRCOM (2010). El estado de la comunicación en España 2010. https://bit.ly/3prJMQZ

DIRCOM (2018). El estado de la comunicación en España 2018. https://bit.ly/2NmVcqL

DIRCOM (2020). Comunicar en tiempos de pandemia. Herramientas y aprendizajes para afrontar el nuevo escenario económico y social propiciado por la COVID-19. https://bit.ly/3c3Gxv3

El Economista.es (2021). Ranking de empresas del sector relaciones públicas y comunicación http://bit.ly/39OdWXS

El Publicista (2019). Las agencias de comunicación y los anunciantes. https://bit.ly/3oc8Z0g

Foncubierta-Rodríguez, M., \& Sánchez-Montero, J. (2019). Hacia la felicidad laboral: Atender motivaciones y eliminar 'temores digitales'. Retos Revista de Ciencias de la Administración y Economía, 9(18), 239-257. https://doi.org/10.17163/ret.n18.2019.04

Gómez-Patiño, M., \& Bandrés-Goldáraz, E. (2014). Las agencias de comunicación aragonesas: su presencia en Internet. En Carmen Marta-Lazo (Ed.), La comunicación digital en Aragón. Antecedentes, estado actual y tendencias. Comunicación Social.

Gómez-Nieto, B. (2011). La comunicación como instrumento de expansión en la pequeña y mediana empresa española: el caso segoviano. Correspondencias y Análisis, 1, 157-172

INE (2020). Indicadores de confianza empresarial. https://bit.ly/3pevfYD

KPMG y CEOE (2020a). La empresa española ante el COVID-19 (marzo). https://bit.ly/3omK8XL KPMG y CEOE (2020b). La empresa española ante el COVID-19 (junio). https://bit.ly/39XITJa

Marta-Lazo, C., Iniesta-Alemán, I., \& Segura Anaya, A. (2018). Cambios en el management de las agencias de publicidad debidos a Internet y las redes sociales. Estudio de caso en Aragón (España). En M. Esteban-Navarro, M. Gómez-Patiño, \& I. Iniesta-Alemán, Comunicación corporativa en Red (pp. 143-162). Egregius.

Mamaqi, X., Miguel, J., \& Olave, P. (2010) The e-DELPHI Method to Test the Importance Competence and Skills: Case of the Lifelong Learning Spanish Trainers. World Academy of Science, Engineering and Technology, 42, 1494-1502. https://bit.ly/3aT4kNj

Marín-Dueñas, P.P. (2015). Comunicación Empresarial en las Pequeñas y Medianas Empresas (PYMES): percepción, conocimiento y actitud. Revista Mediterránea de Comunicación, 6(2), 63-82. https://bit.ly/36839qw

Más de 5000 empresas se adhieren a \#EActíVate por la aceleración económica en solo seis meses. EActíVate. http://bit.ly/3c8Hv9v

Ministerio de Trabajo y Seguridad Social (diciembre, 2020). "Estadística de empresas inscritas en la seguridad social". https://bit.ly/3bMir84

Ministerio de Trabajo y Seguridad Social (diciembre, 2019). "Estadística de empresas inscritas en la seguridad social". https://bit.ly/3o5jgei

Montaña-Blasco, M., Ollé-Castellà, C., \& Lavilla-Raso, M. (2020). Impacto de la pandemia de COVID-19 en el consumo de medios en España. Revista Latina de Comunicación Social, 78, 155-167. https://www.doi.org/10.4185/RLCS-2020-1472

Ravina-Ripoll, R., Marchena-Domínguez, J., \& Montañés Del Río, M. (2019). Happiness Management en la época de la Industria 4.0. RETOS, Revista de Ciencias de la Administración y Economía, 19(18). https://doi.org/10.17163/ret.n18.2019.01

Reglamento (UE) n ${ }^{\circ}$ 651/2014 de la Comisión, de 17 de junio de 2014, por el que se declaran determinadas categorías de ayudas compatibles con el mercado interior en aplicación de los artículos 107 y 108 del Tratado. http://bit.ly/3o4tEDg

Reguant-Álvarez, M., \& Torrado-Fonseca, M. (2016). El método Delphi. REIRE, Revista d'Innovació $i$ Recerca en Educació, 9(1), 87-102. https://doi.org/10.1344/reire2016.9.1916

Scopen (2019). Agency Scope España, 2018/19. https://bit.ly/2MidgSs 